\title{
ON THE AFFINE SURFACE AREA
}

\author{
CARSTEN SCHÜTT
}

(Communicated by William J. Davis)

\begin{abstract}
It is shown that at least two expressions that extend the definition of the affine surface area to all convex bodies coincide.
\end{abstract}

\section{INTRODUCTION}

In the monograph [2] the affine surface area of a convex body $C$ in $\mathbb{R}^{3}$ with sufficiently smooth boundary is introduced by $\int_{\partial C} \kappa(x)^{1 / 4} d \mu(x)$ where $\kappa(x)$ is the Gauss-Kronecker curvature and $\mu$ is the surface measure on $\partial C$. It is then shown that this expression equals

$$
\lim _{\delta \rightarrow 0} \sqrt{\pi} \frac{\operatorname{vol}_{3}(C)-\operatorname{vol}_{3}\left(C_{[\delta]}\right)}{\sqrt{\delta}}
$$

$C_{[\delta]}$ denotes the floating body of $C$ : Every supporting hyperplane of $C_{[\delta]}$ cuts off a set of volume $\delta$ from $C$. It was shown by Leichtweiß [4] that these expressions generalize in the case of higher dimensions to

$$
\begin{gathered}
\int_{\partial C} \kappa(x)^{1 /(n+1)} d \mu(x), \\
\lim _{\delta \rightarrow 0} c_{n} \frac{\operatorname{vol}_{n}(C)-\operatorname{vol}_{n}\left(C_{[\delta]}\right)}{\delta^{2 /(n+1)}}
\end{gathered}
$$

where $c_{n}=2\left(\operatorname{vol}_{n-1}\left(B_{2}^{n-1}(0,1)\right) /(n+1)\right)^{2 /(n+1)}$, provided that $C$ has a $C^{2}$ boundary and $\kappa(x)$ is always positive. Leichtweiß also showed that these expressions are equal. The expressions (1) and (2) do not exist for all convex bodies. Therefore, Leichtweiß suggested the following [5] as the definition for the affine surface area:

$$
\begin{array}{r}
\lim _{\varepsilon \rightarrow 0} \lim _{\delta \rightarrow 0} n c_{n} \delta^{-2 /(n+1)}\left(\operatorname{vol}_{n}\left(C+B_{2}^{n}(0, \varepsilon)\right)-V\left(\left(C+B_{2}^{n}(0, \varepsilon)\right),\right.\right. \\
\left.\left.\ldots,\left(C+B_{2}^{n}(0, \varepsilon)\right),\left(C+B_{2}^{n}(0, \varepsilon)\right)_{[\delta]}\right)\right)
\end{array}
$$

where $V(\ldots)$ denotes the mixed volume.

Received by the editors November 26, 1990.

1991 Mathematics Subject Classification. Primary 52A20.

The author was supported by NSF grant DMS-8902327. 
At the same time Lutwak [8] gave the following as the definition for the affine surface area:

$$
\inf _{L \in S_{C}^{n}}\left\{\left(\int_{\partial B_{2}^{n}} \frac{1}{\rho_{L}(\xi)} d S_{C}(\xi)\right)\left(n \operatorname{vol}_{n}(L)\right)^{1 / n}\right\}^{n /(n+1)}
$$

where $L$ is a star body and $\rho_{L}$ its radius.

Leichtweiß $[6,7]$ proved that $(3)$ is smaller than or equal to $(4)$. It is conjectured that both expressions are equal. In [11] the convex floating body $C_{\delta}$ was studied, i.e., the intersection of all halfspaces $H^{+}$with $\operatorname{vol}_{n}\left(C \cap H^{-}\right)=\delta$. Clearly $C_{\delta}$ exists for all $C$ and $\delta$ and is equal to the floating body whenever it exists. It was shown that

$$
\int_{\partial C} \kappa(x)^{1 /(n+1)} d \mu(x)=\lim _{\delta \rightarrow 0} c_{n} \frac{\operatorname{vol}_{n}(C)-\operatorname{vol}_{n}\left(C_{\delta}\right)}{\delta^{2 /(n+1)}}
$$

where $\kappa(x)$ denotes the generalized Gauss-Kronecker curvature [10, p. 25]. A convex function $\Phi$ on an open subset of $\mathbb{R}^{n}$ is said to be twice differentiable in a generalized sense at $x_{0}$ if there is a linear map $d^{2} \Phi\left(x_{0}\right)$ from $\mathbb{R}^{n}$ into itself so that we have for all $x$ in a neighborhood $U\left(x_{0}\right)$ and all subdifferentials $d \Phi\left(x_{0}\right)$

$$
\left\|d \Phi(x)-d \Phi\left(x_{0}\right)-d^{2} \Phi\left(x_{0}\right)\left(x-x_{0}\right)\right\|_{2} \leq C\left(\left\|x-x_{0}\right\|_{2}\right)\left\|x-x_{0}\right\|_{2},
$$

where $C$ is a function with $\lim _{t \rightarrow 0} C(t)=0$. As curvature radius we take the product of the principal axes of the ellipsoid or ellipsoidal cylinder generated by $d^{2} \Phi\left(x_{0}\right)$. It follows that (3) equals

$$
\lim _{\varepsilon \rightarrow 0} \lim _{\delta \rightarrow 0} c_{n} \delta^{-2 /(n+1)}\left(\operatorname{vol}_{n}\left(C+B_{2}^{n}(0, \varepsilon)\right)-\operatorname{vol}_{n}\left(\left(C+B_{2}^{n}(0, \varepsilon)\right)_{[\delta]}\right)\right) .
$$

We show that the expressions (3) and (5) are equal. Then we show that (5) and, thus, (3) are valuations, a question raised by Leichtweiß [6].

\section{Preliminaries}

The $n$-dimensional volume $\operatorname{vol}_{n}(A)$ of a subset $A$ of $\mathbb{R}^{n}$ is the Lebesgue measure, and the $(n-1)$-dimensional volume $\operatorname{vol}_{n-1}(A)$ is the $(n-1)$-dimensional Hausdorff measure of $A$. The surface measure on the boundary of a convex set is the restriction of the $(n-1)$-dimensional Hausdorff measure to the boundary. We also note that the Hausdorff measure is Borel regular [3]. $B_{2}^{n}(x, r)$ denotes the Euclidean ball with radius $r$ and center $x$ in $\mathbb{R}^{n}$.

A convex surface is almost everywhere twice differentiable in a generalized sense [1]. As a consequence the indicatrix of Dupin exists almost everywhere, and thus we can define a generalized Gauss-Kronecker curvature $\kappa(x)$ that exists almost everywhere [10].

For every $x$ in the boundary $\partial C$ of a convex body $C$ that has a unique normal we define $\Delta(C, x, \delta)$ or $\Delta(x, \delta)$ to be the width of a slice of volume $\delta$ whose defining hyperplane is orthogonal to the normal at $x$. We have [11]

$$
\kappa(x)=\lim _{\delta \rightarrow 0} c_{n} \frac{\Delta(x, \delta)}{\delta^{2 /(n+1)}}
$$

where $c_{n}$ is as in (2). 
For a convex body $C$ in $\mathbb{R}^{n}$ the nearest point projection $q$ from $\mathbb{R}^{n}$ onto $C$ is defined by $\|q(x)-x\|_{2}=\inf _{y \in C}\|y-x\|_{2}$. Let $\widetilde{C}$ be a convex body containing $C$, and let $p$ be the restriction of $q$ to $\partial \widetilde{C}$. Then we have for all Borel subsets $A$ of $\partial \widetilde{C}$ that [3]

$$
\operatorname{vol}_{n-1}(p(A)) \leq \operatorname{vol}_{n-1}(A)
$$

A cap of $C$ at $x$ with height $h$ is denoted by $\operatorname{cap}(C, x, h)$.

\section{The equality of (3) AND (5)}

Proposition 1. The expressions (3) and (5) are equal.

Proposition 1 follows from the next lemma. One has to use that $C_{\delta}$ and $C_{[\delta]}$ coincide whenever $C_{[\delta]}$ exists.

Lemma 2. Let $C$ be a convex body in $\mathbb{R}^{n}$. Then we have

$$
\begin{aligned}
& \lim _{\delta \rightarrow 0} \frac{\operatorname{vol}_{n}(C)-\operatorname{vol}_{n}\left(C_{\delta}\right)}{\delta^{2 /(n+1)}} \\
& \quad=\lim _{\varepsilon \rightarrow 0} \lim _{\delta \rightarrow 0} \delta^{-2 /(n+1)}\left\{\operatorname{vol}_{n}\left(C+B_{2}^{n}(0, \varepsilon)\right)-\operatorname{vol}_{n}\left(\left(C+B_{2}^{n}(0, \varepsilon)\right)_{\delta}\right)\right\} .
\end{aligned}
$$

Proof of Lemma 2. We show first that the right-hand expression of $(8)$ is smaller than the left-hand expression. We have a.e.

$$
\Delta\left(C+B_{2}^{n}(0, \varepsilon), x, \delta\right) \leq \Delta(C, p(x), \delta)
$$

where $p$ is the restriction of the nearest point projection from $\partial\left(C+B_{2}^{n}(0, \varepsilon)\right)$ to $\partial C$. Equation (9) follows from

$$
\operatorname{cap}(C, p(x), h)+\varepsilon N(x) \subseteq \operatorname{cap}\left(C+B_{2}^{n}(0, \varepsilon), x, h\right) .
$$

If a convex body $C$ in $\mathbb{R}^{n}$ contains a Euclidean ball of radius $r$ then

$$
\operatorname{vol}_{n-1}(\partial C) \leq \operatorname{vol}_{n-1}\left(\partial\left(C+B_{2}^{n}(0, \varepsilon)\right)\right) \leq(1+\varepsilon / r)^{n-1} \operatorname{vol}_{n-1}(\partial C)
$$

because $C \subseteq C+B_{2}^{n}(0, \varepsilon) \subseteq(1+\varepsilon / r) C$.

Let $A_{j}$ be measurable subsets of $\partial\left(C+B_{2}^{n}(0, \varepsilon)\right)$ and $a_{j} \geq 0$ so that

$$
\sum_{j=1}^{N} a_{j} \chi_{A_{j}}(x) \leq \lim _{\delta \rightarrow 0} c_{n} \delta^{-2 /(n+1)} \Delta\left(C+B_{2}^{n}(0, \varepsilon), x, \delta\right)
$$

holds almost everywhere and

$$
\begin{aligned}
& (1-\eta) \int_{\partial\left(C+B_{2}^{n}(0, \varepsilon)\right)} \lim _{\delta \rightarrow 0} c_{n} \delta^{-2 /(n+1)} \Delta\left(C+B_{2}^{n}(0, \varepsilon), x, \delta\right) d \mu_{C+B_{2}^{n}(0, \varepsilon)} \\
& \quad \leq \int_{\partial\left(C+B_{2}^{n}(0, \varepsilon)\right)} \sum_{j=1}^{N} a_{j} \chi_{A_{j}} d \mu_{C+B_{2}^{n}(0, \varepsilon)}
\end{aligned}
$$


Then we get

$$
\begin{aligned}
(1- & \eta) \int_{\partial\left(C+B_{2}^{n}(0, \varepsilon)\right)} \lim _{\delta \rightarrow 0} c_{n} \delta^{-2 /(n+1)} \Delta\left(C+B_{2}^{n}(0, \varepsilon), x, \delta\right) d \mu_{C+B_{2}^{n}(0, \varepsilon)} \\
\leq & \sum_{j=1}^{N} a_{j} \mu_{C+B_{2}^{n}(0, \varepsilon)}\left(A_{j}\right) \\
& =\sum_{j=1}^{N} a_{j} \mu_{C}\left(p\left(A_{j}\right)\right)+\sum_{j=1}^{N} a_{j}\left(\mu_{C+B_{2}^{n}(0, \varepsilon)}\left(A_{j}\right)-\mu_{C}\left(p\left(A_{j}\right)\right)\right)
\end{aligned}
$$

By (9) and

$$
\Delta\left(B_{2}^{n}(0, \varepsilon),(\varepsilon, 0, \ldots, 0), \delta\right) \leq \Delta\left(C+B_{2}^{n}(0, \varepsilon), x, \delta\right)
$$

we get that the last expression is smaller than

$$
\begin{aligned}
\int_{\partial C} & \lim _{\delta \rightarrow 0} c_{n} \delta^{-2 /(n+1)} \Delta(C, y, \delta) d \mu_{C}(y) \\
& +\varepsilon^{-(n-1) /(n+1)}\left(\operatorname{vol}_{n-1}\left(\partial\left(C+B_{2}^{n}(0, \varepsilon)\right)\right)-\operatorname{vol}_{n-1}(\partial C)\right) .
\end{aligned}
$$

Because of (10), the second summand can be estimated by

$$
\varepsilon^{-(n-1) /(n+1)}\left((1+\varepsilon / r)^{n-1}-1\right) \operatorname{vol}_{n-1}(\partial C) .
$$

Therefore, we get altogether

$$
\begin{aligned}
\lim _{\varepsilon \rightarrow 0} \sup & \int_{\partial\left(C+B_{2}^{n}(0, \varepsilon)\right)} \lim _{\delta \rightarrow 0} c_{n} \delta^{-2 /(n+1)} \Delta\left(C+B_{2}^{n}(0, \varepsilon), x, \delta\right) d \mu_{C+B_{2}^{n}(0, \varepsilon)} \\
\leq & \int_{\partial C} \lim _{\delta \rightarrow 0} c_{n} \delta^{-2 /(n+1)} \Delta(C, y, \delta) d \mu_{C} .
\end{aligned}
$$

In view of (6) we may plug in $\kappa(x)$.

In order to show that the right-hand side of $(8)$ is larger than the left-hand side we require a lemma.

Lemma 3. Let $x \in \partial\left(C+B_{2}^{n}(0, \varepsilon)\right)$, and suppose that the indicatrix of Dupin at $p(x) \in \partial C$ is an ellipsoid with radius $R=\left(R_{1}, \ldots, R_{n-1}\right)$. Then we have

$$
\kappa\left(\partial\left(C+B_{2}^{n}(0, \varepsilon)\right), x\right)=\prod_{i=1}^{n-1}\left(R_{i}(p(x))+\varepsilon\right)^{-1} .
$$

The set $\{y \in \partial C \mid \kappa(y)>0\}$ is measurable since $\kappa(y)^{1 /(n+1)} \in L^{1}(\partial C)$ [11]. Since the Hausdorff measure is Borel regular, there is a subset $A$ of $\{y \in$ $\partial C \mid \kappa(y)>0\}$ that is a Borel set having the same measure. By Lemma 3 we obtain

$$
\begin{aligned}
& \int_{\partial\left(C+B_{2}^{n}(0, \varepsilon)\right)} \kappa(x)^{1 /(n+1)} d \mu_{C+B_{2}^{n}(0, \varepsilon)} \\
& \quad \geq \int_{p^{-1}(A)} \prod_{i=1}^{n-1}\left(R_{i}(p(x))+\varepsilon\right)^{-1 /(n+1)} d \mu_{C+B_{2}^{n}(0, \varepsilon)}
\end{aligned}
$$


As above we get that the last expression is larger than or equal to

$$
\int_{A} \prod_{i=1}^{n-1}\left(R_{i}(y)+\varepsilon\right)^{-1 /(n+1)} d \mu_{C}
$$

Applying Fatou's lemma we get

$$
\liminf _{\varepsilon \rightarrow 0} \int_{\partial\left(C+B_{2}^{n}(0, \varepsilon)\right)} \kappa(x)^{1 /(n+1)} d \mu_{C+B_{2}^{n}(0, \varepsilon)} \geq \int_{\partial C} \kappa(y)^{1 /(n+1)} d \mu_{C} .
$$

\section{The AFFine surface AREA is a VAluation}

A map $T$ from the family of convex bodies into $\mathbb{R}$ is called a valuation if

$$
T(K \cup L)+T(K \cap L)=T(K)+T(L)
$$

whenever $K \cup L$ is convex.

Proposition 4. The affine surface area is a valuation.

Lemma 5. Let $K$ and $L$ be convex bodies in $\mathbb{R}^{n}$, and suppose that $K \cup L$ is a convex body. Then we have for all $x \in \partial K \cap \partial L$ where all the curvatures $\kappa_{K \cup L}, \kappa_{K \cap L}, \kappa_{K}$, and $\kappa_{L}$ exist that

$$
\begin{aligned}
& \kappa_{K \cup L}(x)=\min \left\{\kappa_{K}(x), \kappa_{L}(x)\right\}, \\
& \kappa_{K \cap L}(x)=\max \left\{\kappa_{K}(x), \kappa_{L}(x)\right\} .
\end{aligned}
$$

Please note that the set where one of the curvatures does not exist is a null set [10].

For the proof of Lemma 5 we only have to observe that the indicatrix of Dupin of $K \cup L$ at $x$ is the union of those of $K$ and $L$ at $x$. Moreover, the indicatrix of $K \cap L$ at $x$ is the intersection of those of $K$ and $L$. Then one uses that the intersection or union of two ellipsoids is again an ellipsoid if and only if one ellipsoid is contained in the other.

Proof of Proposition 4. The affine surface area of a convex body $M$ equals $\int_{\partial M} \kappa_{M}(x)^{1 /(n+1)} d \mu_{M}$. We apply this formula to the bodies $K \cup L, K \cap L, K$, and $L$, and decompose the surfaces

$$
\begin{aligned}
\partial(K \cup L) & =\{\partial K \cap \partial L\} \cup\left\{\partial K \cap L^{\mathrm{c}}\right\} \cap\left\{\partial L \cap K^{\mathrm{c}}\right\}, \\
\partial(K \cap L) & =\{\partial K \cap \partial L\} \cup\{\partial K \cap \stackrel{\circ}{L}\} \cup\{\partial L \cap \stackrel{\circ}{K}\}, \\
\partial K & =\{\partial K \cap \partial L\} \cup\left\{\partial K \cap L^{\mathrm{c}}\right\} \cup\{\partial K \cap \stackrel{\circ}{L}\}, \\
\partial L & =\{\partial K \cap \partial L\} \cup\left\{\partial L \cap K^{\mathrm{c}}\right\} \cup\{\partial L \cap \stackrel{\circ}{K}\},
\end{aligned}
$$

where $K^{\mathrm{c}}$ is the complement of $K$ and $\stackrel{\circ}{K}$ is the interior of $K$.

Since all sets (except possibly $\partial K \cap \partial L$ ) are open subsets of $\partial K, \partial L$, $\partial(K \cap L)$, and $\partial(K \cup L)$ and since the curvature is a local invariant, the 
integrals over those sets cancel out. It remains to show

$$
\begin{gathered}
\int_{\partial K \cap \partial L} \kappa_{K \cup L}(x) d \mu_{K \cup L}+\int_{\partial K \cap \partial L} \kappa_{K \cap L}(x) d \mu_{K \cap L} \\
=\int_{\partial K \cap \partial L} \kappa_{K}(x) d \mu+\int_{\partial K \cap \partial L} \kappa_{L}(x) d \mu .
\end{gathered}
$$

This follows from Lemma 5.

\section{REFERENCES}

1. V. Bangert, Analytische Eigenschaften konvexer Funktionen auf Riemannschen Mannigfaltigkeiten, J. Reine Angew. Math. 307 (1979), 309-324.

2. W. Blaschke, Vorlesungen über Differentialgeometrie. II, Springer-Verlag, Berlin and New York, 1923.

3. H. Federer, Geometric measure theory, Springer-Verlag, Berlin and New York, 1969.

4. K. Leichtweiß, Über eine Formel Blaschkes zur Affinoberfläche, Studia Sci. Math. Hungar. 21 (1986), 453-474.

5. __ Zur Affinoberfläche konvexer Körper, Manuscripta Math. 56 (1986), 429-464.

6. __ paper presented at the conference "Konvexgeometrie" in Oberwolfach, 1990.

7. _ Bemerkungen zur Definition einer erweiterten Affinoberfläche von E. Lutwak, Manuscripta Math. 65 (1989), 181-197.

8. E. Lutwak, Extended affine surface area, Adv. in Math. 85 (1991), 39-68.

9. P. McMullen and R. Schneider, Valuations on convex bodies, Convexity and Its Applications (P. M. Gruber and J. M. Wills, eds.), Birkhäuser Verlag, Boston, Basel, and Berlin, 1983.

10. R. Schneider, Boundary structure and curvature of convex bodies, Proc. Geom. Sympos. Siegen (J. Tölke and J. M. Wills, eds.), Birkhäuser, Boston, Basel, and Berlin, 1978, pp. 13-59.

11. C. Schütt and E. Werner, The convex floating body, Math. Scand. 66 (1990), 275-290.

Department of Mathematics, Oklahoma State University, Stillwater, Oklahoma 74078

Current address: Mathematisches Seminar, Christian Albrechts Universität, 23 Kiel, Germany 